\title{
The role of phase space geometry in Heisenberg's uncertainty relation
}

\author{
Charis Anastopoulos * \\ Spinoza Instituut, Leuvenlaan 4, \\ 3584HE Utrecht, The Netherlands \\ and \\ Ntina Savvidou ${ }^{\dagger}$ \\ Theoretical Physics Group, \\ Imperial College, SW7 2BZ London, \\ UK
}

October 26, 2018

\begin{abstract}
Aiming towards a geometric description of quantum theory, we study the coherent states-induced metric on the phase space, which provides a geometric formulation of the Heisenberg uncertainty relations (both the position-momentum and the time-energy ones). The metric also distinguishes the original uncertainty relations of Heisenberg from the ones that are obtained from non-commutativity of operators. Conversely, the uncertainty relations can be written in terms of this metric only, hence they can be formulated for any physical system, including ones with non-trivial phase space. Moreover, the metric is a key ingredient of the probability structure of continuous-time histories on phase space. This fact allows a simple new proof the impossibility of the physical manifestation of the quantum Zeno and anti-Zeno paradoxes. Finally, we construct the coherent states for a spinless relativistic particle, as a non-trivial example by which we demonstrate our results.
\end{abstract}

*anastop@phys.uu.nl

†ntina@ic.ac.uk 


\section{Introduction}

In the present paper we focus on the geometric description of quantum theory, through the study of coherent states. Of key importance is a metric $d s^{2}$ on phase space, which is induced by the coherent states construction. Remarkably, this metric includes both types of uncertainty in its structure, but in a distinct mathematical fashion. Moreover, the Heisenberg uncertainty relations can be written straightforwardly in terms of the metric as $\delta s^{2} \sim 1$. This is an important result, because it allows the formulation of the Heisenberg uncertainty relations for any physical system, in particular the ones with non-trivial phase space.

The uncertainty relations are a key component of quantum theory that signifies a fundamental inability to simultaneously determine the values of the fundamental physical quantities with arbitrary accuracy. There exist two different versions of the uncertainty relations. The original version is due to Heisenberg [1]; it refers to measurements of individual quantum systems and does not employ the mathematical formalism of quantum theory, but derives the uncertainty relations as a consequence of wave-particle duality. The second version employs the formalism of quantum theory and derives the uncertainty relation as a consequence of the non-commutativity of operators. However, this derivation does not refer to individual quantum systems, as the uncertainties are, essentially, the mean deviations of measurements in a statistical ensemble.

This distinction is not usually emphasised, mainly because the statistical uncertainty relation, which is mathematically concrete, can be viewed as a consequence of the Heisenberg's uncertainty relations. However, the distinction persists not only because of the different context of the two types of uncertainty, but also because the time-energy uncertainty relation does not have an operator analogue ${ }^{1}$.

Our discussion is closely related to the consistent histories description of quantum theory $[4,5,6,7,8]$. We show that the probability assignment for continuous-time histories on phase space depends explicitly on the metric $d s^{2}$, a fact that highlights the geometric character of quantum probability. This is intended to provide a starting point for a procedure of quantisation in terms of histories that will be fully geometric. Moreover, the relation of the metric to the uncertainty relation allows us to demonstrate in a simple manner that the quantum Zeno effect [9] cannot be physically manifested.

The structure of this paper is the following. In section 2 we give the relevant background, namely the formalism of quantum mechanical histories and the geometry of coherent states. The main results of the paper are in section 3 . We show how the probabilities for continuous-time histories have geometrical origin, the relation of the metric to the uncertainty relations and their consequences.

\footnotetext{
${ }^{1}$ The reason for this is the inability to define a time operator. However, one can derive some operator versions of the time-energy uncertainty relation by considering some quantum mechanical variable as a clock that measures time (see [2] for a recent review). However, no quantum mechanical clock is guaranteed to always move forward in time [3].
} 
In section 4 we give an explicit example for our results, namely we construct the coherent states for a spinless relativistic particle, identify the corresponding geometry and consequently write the relativistic version of the Heisenberg uncertainty relations.

\section{Background}

\subsection{Quantum mechanical histories}

The histories formalism describes quantum mechanical systems in terms of the properties that refer to more than one moment of time. These are the histories. In this sense the relation of the standard quantum mechanical formalism to the histories one is analogous to the relation of Hamilton's formulation vs the Lagrangian action one in classical mechanics [10, 11]: Hamilton's formulation is based on the properties of a system at a single moment of time and studies their evolution, while the action principle starts with paths (histories) and seeks which one of them are realisable. A similar distinction can be made in the context of classical probability theory: the evolution of single-time properties is effected by the Fokker-Planck equation for a probability distributions, while the theory of stochastic processes deals with the probabilities of paths. The formalism of quantum mechanical histories can be developed in such a way as to explicitly denote the analogy with both classical mechanics [10] and stochastic processes $[12,13]$.

The histories description arose as part of a particular interpretation of quantum theory, namely consistent or decoherent histories $[4,5,6,7,8]$. This interpretation considers quantum theory as a theory that describes individual closed systems, in contrast with the Copenhagen interpretation that refers to systems in the context of a measurement procedure and interprets the probabilities in terms of ensembles of quantum systems.

A key feature of the histories description is that the probabilities for histories do not satisfy the additivity condition of Kolmogorov. If $\alpha$ is a proposition about a history and $\bar{\alpha}$ its negation, it is not necessary that $p(\alpha)+p(\bar{\alpha})=1$. Hence even if we can ascertain that $p(\alpha)=1$, we cannot preclude the possibility that $\bar{\alpha}$ can also take place. This would not be a problem for a theory that cares to describe only measurement outcomes as the measurement of history $\alpha$ and the measurement of history $\bar{\alpha}$ refer to different experimental setups. However, it is quite problematic for the predictability of any theory that describes individual systems. The consistent or decoherent histories interpretation avoids this problem by postulating that one can use probabilities to make inference only when one works within particular sets of histories within which the probabilities are additive. Such sets are known as consistent sets and they are obtained by a decoherence condition.

The consistent histories interpretation has its points of contention, namely 
that one can make inferences within different consistent sets and conclude contrary properties [17] — without, however, arriving at a logical contradiction [18]. Like all interpretations of quantum theory that aim to describe individual systems, the consistent histories has to accept that properties assigned to systems are contextual [19].

However, the history formalism exhibits a pluralism and can be employed without a commitment to the consistent histories interpretation. In the present paper we will assume a convenient Copenhagen stance, which states that probabilities refer to experiments and are determined by measurements of ensembles of quantum systems.

A history is a time-ordered sequence of properties of a quantum system. A single time property is determined by a projection operator, so a history is an ordered set of projectors labelled by time

$$
\alpha \rightarrow\left(\hat{\alpha}_{t_{1}}, \ldots, \hat{\alpha}_{t_{n}}\right)
$$

To each history one can assign the operator $\hat{C}_{\alpha}$ defined by

$$
\alpha \rightarrow \hat{C}_{\alpha}=\hat{\alpha}_{t_{1}}\left(t_{1}\right) \ldots \hat{\alpha}_{t_{n}}\left(t_{n}\right),
$$

where $\hat{\alpha}(t)$ refers to the Heisenberg picture projector

$$
\hat{\alpha}_{t}(t)=e^{i \hat{H} t} \hat{\alpha}_{t} e^{-i \hat{H} t} .
$$

Note the distinct appearance of time $t$ as an argument of the temporal ordering of projectors and as the parameter of Heisenberg time evolution [10, 20]. Here $\alpha_{t}$ is a Schrödinger picture operator at a fixed time $t$; in general it is different from the time-parameter $t$ that appears in $e^{-i \hat{H} t}$. This is a basic feature of the temporal structure of histories quantum theory as has been identified in previous work of one of us $[10,11]$. In particular, we employ the word time to denote two different physical concepts in physical theories. The first is the notion of causality in spacetime, i.e. the designation of whether one event is prior or later to another. The other is the notion of evolution, i.e. time as a parameter in the equations of motion that measures how much a physical system has changed from some initial instant. In classical mechanics and - to a large extent - in quantum theory this distinction is not essential: the timeparameter of Hamilton's or Heisenberg's time evolution is usually thought of as incorporating both notions of time.

However, the distinction is natural in the histories framework and may be essential in general relativity $[11,44]$ as any attempt to quantise this theory needs to deal with the "problem of time" 2 (see $[14,15]$ for a review).

\footnotetext{
${ }^{2}$ The problem of time amounts to the following. In General Relativity the Hamiltonian vanishes due to constraints. How can we reconcile this with the causal description necessary for any physical theory? This is a problem already at the classical level, but at the quantum level it is even more acute. Because standard quantum theory necessitates a background causal structure in order to make sense, while in General Relativity the causal structure is itself a dynamical quantity.
} 
From the operators (2.2) one can construct the decoherence functional. This is a complex valued function of pairs of histories

$$
(\alpha, \beta) \rightarrow d(\alpha, \beta)=\operatorname{Tr}\left(\hat{C}_{\alpha}^{\dagger} \hat{\rho}_{0} \hat{C}_{\beta}\right) .
$$

Here $\hat{\rho}_{0}$ is the density matrix at time $t=0$. This object contains all information related to the state of the system and the dynamics. Its diagonal elements are the probabilities $p(\alpha)$ for the histories,

$$
d(\alpha, \alpha)=p(\alpha) .
$$

Its off-diagonal elements have an interpretation in terms of geometric phases $[21,22,23]$. In the generic case $d(\alpha, \beta)$ is a complex number $r e^{i \theta}$. The phase $e^{i \theta}$ is a special case of the Pancharatnam phase $[24,25]$ and is, in principle, measurable [12]. The modulus $r$ can also be operationally determined, but is less interesting ${ }^{3}$.

It is easier to see the appearance of a geometric phase in the off-diagonal elements of the decoherence functional in the particular case of very fine-grained histories, i.e. histories consisting of one-dimensional projectors [16]. To this end we consider two histories

$\alpha=\left(\left|\psi_{t_{1}}\right\rangle\left\langle\psi_{t_{1}}|, \ldots| \psi_{t_{n}}\right\rangle\left\langle\psi_{t_{n}}\right|\right)$ and $\beta=\left(\left|\psi_{t_{1}^{\prime}}^{\prime}\right\rangle\left\langle\psi_{t_{1}^{\prime}}^{\prime}|, \ldots| \psi_{t_{m}^{\prime}}\right\rangle\left\langle\psi_{t_{m}^{\prime}}\right|\right)$ and we construct the decoherence functional.

For simplicity, we take $\hat{H}=0$ and $\hat{\rho}_{0}$ corresponding to a pure state $\psi_{0}$. From (2.4) we get

$$
d(\alpha, \beta)=\left\langle\psi_{t_{n}} \mid \psi_{t_{n-1}}\right\rangle \ldots\left\langle\psi_{t_{1}} \mid \psi_{0}\right\rangle\left\langle\psi_{0} \mid \psi_{t_{1}^{\prime}}^{\prime}\right\rangle \ldots\left\langle\psi_{t_{m-1}^{\prime}}^{\prime} \mid \psi_{t_{m}^{\prime}}^{\prime}\right\rangle
$$

The right-hand side of this equation is known as the $n+m+1$ Bargmann invariant $t^{4}$. Moreover, if we take the continuum limit defined by $\delta t=\sup \left\{\mid t_{i}-\right.$ $\left.t_{i-1}|,| t_{j}^{\prime}-t_{j-1}^{\prime} \mid\right\}$ going to zero, the decoherence functional reads

$$
d(\alpha, \beta)=e^{\left(\sum_{i=1}^{n}\left\langle\psi_{t_{i}} \mid \psi_{t_{i}}-\psi_{t_{i-1}}\right\rangle-\sum_{i=0}^{m}\left\langle\psi_{t_{i}^{\prime}} \mid \psi_{t_{i}^{\prime}}-\psi_{t_{i-1}^{\prime}}\right\rangle\right)}+O\left(\delta t^{2}\right) .
$$

This converges to

$$
d(\alpha, \beta)=e^{i \int_{C} A},
$$

where $A$ is an one-form defined over the projective Hilbert space $P \mathcal{H}$

$$
i A=\langle\psi \mid d \psi\rangle,
$$

\footnotetext{
${ }^{3}$ The decoherence functional plays an additional role. If in place of the projectors in equations (2.3) and (2.4) we insert a self-adjoint operator $\hat{A}$ then the values of the decoherence functional are the mixed time-ordered and anti-time-ordered correlation functions of $\hat{A}$. Thus the decoherence functional can be identified $[26,12]$ as the object that contains all temporal correlation functions of the theory: it is known as the closed-time-path generating functional and was introduced by Schwinger [28].

${ }^{4}$ The invariance it refers to is with respect to the group $U(1)^{n+m+1}$ in its action $\left|\psi_{t}\right\rangle \rightarrow$ $e^{i \alpha}\left|\psi_{t}\right\rangle$.
} 
with $d$ the exterior derivative on $P \mathcal{H}$ and $C$ is the closed loop formed by the combination of the path $\psi(\cdot)$ and $\psi^{\prime}(\cdot)$. In fact, $A$ is a connection one-form, known as the Berry connection.

From equation (2.7) it is clear that $d(\alpha, \beta)$ equals the holonomy of the Berry connection over $C$ : this is the well-known Berry phase associated to the path [23]. Its value is invariant under the gauge $U(1)$ transformation (we suppress the primes for simplicity)

$$
\left|\psi_{t}\right\rangle \rightarrow e^{i \theta(t)}\left|\psi_{t}\right\rangle .
$$

\subsection{Phase space in quantum theory}

The expressions (2.6) above refer, in general, to histories on a Hilbert space $\mathcal{H}$. In what follows, we will focus on histories that correspond to points of the classical phase space and we shall translate all structures we encountered so far in that context.

The reason for this is primarily interpretational. In particular [27, 12] it has been proposed in $[27,12]$ that quantum theory can be formulated solely in the classical phase space and without making any reference to the quantum mechanical Hilbert space. In this construction the fundamental observables are commutative and the quantum behavior is contained in the relative phases between histories. This information is fully encoded in the decoherence functional. It was shown that all predictions of quantum theory can thus be derived, including the predictions associated to Bell's theorem.

The key result from this is the important statement that non-commutativity is not an essential feature of quantum theory, in the sense that predictions identical to the ones of tandard quantum theory can be obtained from an axiomatic scheme, which is fundamentally based on commutative variables. This fact is rather important as it provides a new perspective towards possible interpretations of quantum theory (see [12] for a summary).

In this approach a reconstruction theorem was proven [12]. If, in particular, we start from a theory that satisfies the history axioms on phase space, we can uniquely write a standard quantum theory on a Hilbert space, in such a way that the statistical predictions are identical. The translation between the phase space and the Hilbert space concepts is effected by means of coherent states. To their description we shall now turn.

\subsection{Coherent states and their geometry}

Hilbert space geometry. In most approaches to quantum theory the rich geometry of the Hilbert space is not particularly emphasised, even if this geometry is a fundamental part of quantum theory. The reason for this is that the formalism of quantum theory, as it was originally developed, does not highlight the geometric structure of the theory. However, this structure is present and is manifested in the appearance of the geometric phases. 
It is well known that the inner product of a complex Hilbert space $\mathcal{H}$ defines a metric and a symplectic form, from its real and imaginary part respectively. If we remove the null vector from $\mathcal{H}$ the resulting space is a total space of a bundle, with base space the projective Hilbert space, fiber the complex numbers C and projection map $\pi:|\psi\rangle \rightarrow(\langle\psi \mid \psi\rangle)^{-1}|\psi\rangle\langle\psi|$. This is clearly a line bundle, which is often called the Hopf bundle, and denoted as $\mathbf{H}$.

Due to the bundle structure the inner product on $H$ induces a metric, a connection and a symplectic form [29] on $P \mathcal{H}$. In terms of a representative normalised vector $|\psi\rangle$,

$$
\begin{array}{r}
d s^{2}=\langle d \psi \mid d \psi\rangle-|\langle\psi \mid d \psi\rangle|^{2}, \\
i A=\langle\psi \mid d \psi\rangle, \\
\Omega=d A .
\end{array}
$$

The connection one-form $A$ is the Berry connection we encountered earlier, the symplectic form is the curvature of $A$ and the metric is known as the FubiniStudy metric. Note that a transformation $|\psi\rangle \rightarrow e^{i \theta}|\psi\rangle$ affects the connection one-form

$$
A \rightarrow A+d \theta .
$$

General coherent states. The coherent states form a bridge between quantum theory and classical symplectic mechanics. A set of coherent states is defined as a map from a manifold $\Gamma$ to the projective Hilbert space $P \mathcal{H}$,

$$
i: z \in \Gamma \rightarrow|z\rangle\langle z| \in P H .
$$

Note that $|z\rangle$ refers to a representative (up to a phase) normalised vector on the complex Hilbert space. Part of the definition of coherent states is often the requirement that the set of vectors $|z\rangle$ forms a resolution of the unity, i.e. given a measure $\mu$ on $\Gamma$, we have

$$
1=\int d \mu(z)|z\rangle\langle z|
$$

Coherent states are often constructed by means of group representations. If a group has a unitary representation $\hat{U}(g), g \in G$ on a Hilbert space $H$, then we can construct the vectors $\hat{U}(g)|0\rangle$, where $|0\rangle$ is a reference vector. The usual choice for $|0\rangle$ is either the minimum energy state or a vector that is invariant under the maximal compact subgroup of $G$. Then we define the equivalence relation on $G$ as follows:

$g \sim g^{\prime}$ if there exists $e^{i \theta} \in U(1)$ such that $\hat{U}(g)|0\rangle=e^{i \theta} \hat{U}\left(g^{\prime}\right)|0\rangle$.

Defining the manifold $\Gamma=G / \sim$, the map

$$
[g]=z \in \Gamma \rightarrow \hat{U}(g)|0\rangle\langle 0| \hat{U}^{\dagger}(g)
$$


defines a set of coherent states $|z\rangle$. Furthermore, this set does possess a resolution of the unity.

The map $i: \Gamma \rightarrow P \mathcal{H}$ can be employed to pull-back the geometrical objects defined on $P \mathcal{H} \mathrm{s}$ to $\Gamma$. In particular we can define on $\Gamma$, the pull-back bundle $i^{*} \mathbf{H}$, the metric, the connection and its curvature form

$$
\begin{array}{r}
d s_{\Gamma}^{2}=\langle d z \mid d z\rangle-|\langle z \mid d z\rangle|^{2}, \\
i A_{\Gamma}=\langle z \mid d z\rangle, \\
\Omega_{\Gamma}=d A_{\Gamma} .
\end{array}
$$

The two-form $\Omega_{\Gamma}$ can, in general, be degenerate: if it is not, $\Gamma$ has the structure of a symplectic manifold. Then the Liouville form $\Omega \wedge \ldots \wedge \Omega$ defines a measure on $\Gamma$ and makes possible the existence of a resolution of a unity. We want to emphasise that the bundle structure, the U(1) connection and the metric on $\Gamma$ are defined, irrespective of whether there exists a resolution of the unity or not.

Our main motivation is to study the quantum structure of histories on phase space and the coherent states are the key feature, which translates the structures of standard quantum theory into geometric objects on the classical phase space.

The idea that the classical phase space is a fundamental ingredient of quantum theory is very intriguing. However the study of coherent state histories is interesting by itself, because it allows one to reproduce all quantum mechanical predictions. In order to demonstrate this feature of coherent states we need to recall two basic results.

Firstly, the function that gives the inner product between two coherent states $\left\langle z \mid z^{\prime}\right\rangle$ contains sufficient information to construct the corresponding Hilbert space. Quite simply the inner product $\left\langle z \mid z^{\prime}\right\rangle$ defines the matrix elements of a projector $E$ on the Hilbert space $L^{2}(\Gamma)$ : the range of this projector is the Hilbert space of the theory [30].

Secondly, most operators on $\mathcal{H}$ can be written in terms of functions on $\Gamma$ as

$$
\hat{A}=\int d \mu(z) f_{A}(z)|z\rangle\langle z|,
$$

provided there exists a resolution of the unity. Hence if we construct the decoherence functional on coherent states, we can reproduce its values for any history.

The standard coherent states. The most usual case of coherent states are the ones associated with the Weyl group. Let us consider for simplicity the one-dimensional case, in which the generators are $\hat{x}, \hat{p}, \hat{1}$. We shall use the Schrödinger representation, in which the vectors are square integrable functions of $x$ and the elements of the Weyl group act as

$$
\begin{array}{r}
e^{-i \hat{p} q} \psi(x)=\psi(x-q) \\
e^{-i \hat{x} p} \psi(x)=e^{-i p x} \psi(x) .
\end{array}
$$


We choose a reference state $\psi_{0}(x)$ with vanishing expectation value of $\hat{x}$ and $\hat{p}$. Then for $z=(q, p)$ the coherent states read

$$
\psi_{z}(x)=e^{i p x} \psi_{0}(x-q) .
$$

We can then show that

$$
\begin{aligned}
A & =p d q \\
d s^{2} & =(\Delta p)^{2} d q^{2}+(\Delta q)^{2} d p^{2}+2 C_{p q} d p d q
\end{aligned}
$$

where $\Delta q$ and $\Delta p$ are the standard quantum mechanical uncertainties associated to the coherent state $|z\rangle$ (now written in the Dirac notation) and $C_{p q}=\frac{1}{2}\langle z| \hat{x} \hat{p}+$ $\hat{p} \hat{x}|z\rangle-2\langle z|\hat{x}| z\rangle\langle z|\hat{p}| z\rangle$ is the correlation between position and momentum. Note that neither the uncertainties nor the correlations depend on $z$ and can therefore be defined with respect to the reference vector $\psi_{0}$.

We usually choose the reference vector $\psi_{0}$, so that $C_{p q}=0$. The standard choice is

$$
\psi_{0}(x)=\frac{1}{\left(\pi \sigma^{2}\right)^{1 / 4}} e^{-\frac{x^{2}}{2 \sigma^{2}}} .
$$

In what follows, we shall show the important role of the geometry of coherent states in the probability structure of continuous-time histories.

\section{Phase space histories}

We will now study the decoherence functional for phase space histories. For this purpose it is sufficient to examine its values for the finest-grained histories, as any history can be obtained by coarse-graining on phase space [31, 12]. The classical phase space is a symplectic manifold $\Gamma$ of dimension $n$, whose points will be denoted by $z$.

\subsection{Phase space histories with zero Hamiltonian}

\subsubsection{The expansion of the propagator}

We shall first consider the case of trivial dynamics, i.e. $\hat{H}=0$. From equation (2.6) we see that in order to construct the decoherence functional we need to compute the inner product $\left\langle z \mid z^{\prime}\right\rangle$.

In order to study continuous-time histories we need to consider the case that $z^{\prime}$ is infinitesimally close to $z: z^{\prime}=z+\delta z$. We then expand the inner product in powers of $\delta z$ and keep terms of order $(\delta z)^{2}$

$$
\langle z \mid z+\delta z\rangle=1+\left\langle z \mid \partial_{i} z\right\rangle \delta z^{i}+\frac{1}{2}\left\langle z \mid \partial_{i} \partial_{j} z\right\rangle \delta z^{i} \delta z^{j}+O\left(\delta z^{3}\right) .
$$

¿From equations (2.18-2.19) we see that the components of the connection form and the metric on $\Gamma$ read 


$$
\begin{array}{r}
i A_{i}(z)=\left\langle z \mid \partial_{i} z\right\rangle, \\
g_{i j}(z)=\left\langle\partial_{i} z \mid \partial_{j} z\right\rangle+A_{i}(z) A_{j}(z),
\end{array}
$$

where the indices $i, j$ run from 1 to $n$.

Hence,

$$
\left\langle z \mid \partial_{i} \partial_{j} z\right\rangle=-g_{i j}(z)-A_{i}(z) A_{j}(z)+i \partial_{i} A_{j}(z) .
$$

This implies that

$$
\langle z \mid z+\delta z\rangle=\exp \left(i A_{i}\left(z+\frac{\delta z}{2}\right) \delta z^{i}-\frac{1}{2} g_{i j} \delta z^{i} \delta z^{i}\right)+O\left(\delta z^{3}\right) .
$$

We now consider a pair of histories $\alpha$ (corresponding to $r \rightarrow\left|z_{r}\right\rangle$ ) and $\beta$ (corresponding to $r^{\prime} \rightarrow\left|z_{r^{\prime}}^{\prime}\right\rangle$ ). The integers $r$ and $r^{\prime}$ denote the time instants and take values $r=1, \ldots n, r^{\prime}=1 \ldots m$. Furthermore, we assume that the initial point on phase space is given by $\left|z_{0}\right\rangle$ and that $z_{n}=z_{m}^{\prime}$.

We construct the loop $a \rightarrow\left|z_{a}\right\rangle$, with $a=0, n+m$ from the combination of the two previous paths, namely

$$
z_{a}=\left\{\begin{array}{cc}
z_{r}, & a=1, \ldots n \\
z_{a-n}^{\prime}, & a=n+1, n+m \\
z_{0}, & a=0
\end{array}\right.
$$

From equation (2.6) we write the decoherence functional

$$
d(\alpha, \beta)=\prod_{a}\left\langle z_{a} \mid z_{a}+\delta z_{a}\right\rangle,
$$

where $\delta z_{a}=z_{a+1}-z_{a}$ and where we have refrained from writing explicitly a time parameter. Finally,

$$
d(\alpha, \beta)=\exp \left(i \sum_{a} A\left(z_{a}+\frac{\delta z_{a}}{2}\right) \delta z_{a}-\frac{1}{2} \sum_{a} \delta s_{a}^{2}\right)+O\left(\delta z^{3}\right) .
$$

If $\left|\delta z_{a}^{i}\right|<\epsilon$ and we subsequently let $\epsilon \rightarrow 0^{5}$, the expression (3.8) converges to $\exp \left(i \int_{C} A\right)$, as we showed in section 1 .

We should note here that for any fine-grained history $\alpha$, the probability $d(\alpha, \alpha) \rightarrow 1$ in the continuum limit. This seems to imply that a continuous measurement of whether the quantum system follows a path forces the system to actually follow the path.

This behaviour is reminiscent of the quantum Zeno effect [9], which refers to the following behaviour. When we monitor continuously a quantum system in order to see if a transition has occurred, we inhibit the transition so that the probability that the system remains in the initial state is equal to one. In the present case an anti-Zeno effect [32] is manifested, a probability equal to one is assigned to a general path rather than to the preservation of a state in time.

\footnotetext{
${ }^{5}$ This means that the paths are continuous and their variation is bounded.
} 


\subsubsection{Uncertainty relation}

As we showed in section 1.4, the phase space metric for the case of the Weyl group is written

$$
\delta s^{2}=(\Delta p)^{2} \delta q^{2}+(\Delta q)^{2} \delta p^{2}+2 C_{p q} \delta p \delta q .
$$

In this equation, there exist two types of "uncertainties" for physical quantities, namely $\delta$ and $\Delta$. Their physical interpretations are distinct and for this reason we will elaborate on the meaning of the Heisenberg uncertainty relations.

When Heisenberg first wrote the uncertainty relations, he was referring to the uncertainty in the measurement of position and momentum of an individual quantum system. He did not make use of the formalism of quantum mechanics; the uncertainty relation was a consequence of solely the wave-particle duality. It is interesting to recall his argument: In order to measure the position of a particle, we need to employ photons (or electrons) of some wavelength $\lambda$. There will then be an inaccuracy in the measurement of the order of $\delta x \sim \lambda$. In order to measure momentum with the same source of photons, we cannot have higher accuracy than the momentum of a single photon, so $\delta p \sim \frac{\hbar}{\lambda}$. We altogether have an uncertainty $\delta x \delta p \sim \hbar$.

On the other hand, starting from the formalism of quantum mechanics one can derive another uncertainty relation

$$
\Delta q \Delta p \geq \frac{\hbar}{2},
$$

as a consequence of the non-commutativity of the operators. Here $\Delta q$ is defined as

$$
(\Delta q)^{2}=\left\langle\psi\left|\hat{q}^{2}\right| \psi\right\rangle-(\langle\psi|\hat{q}| \psi\rangle)^{2},
$$

and similarly is $\Delta p$ defined. These quantities are interpreted as mean deviations of the distributions of position and momenta respectively, hence they are statistical objects. Each of them refers to a different type of measurement, hence a different experimental set-up; they do not refer to individual systems. In fact, they can be viewed as features of the quantum state $|\psi\rangle$, through which they are defined. In what follows we shall refer to the first uncertainty principle as the Heisenberg uncertainty principle and to the second as the statistical uncertainty principle.

A widespread belief is that the uncertainty relations proves that the phase space properties of physical systems are not simultaneously definable. This is not true for either of them. Both uncertainty relations make explicitly reference to measurements and hence refer to open systems. The only demonstration of such non-definability comes in the wake of the Kochen-Specker theorem [35], but this necessitates the dubious assumption that the quantum mechanical formalism (operators and states) refers to individual systems rather than ensembles. 
Clearly in the Copenhagen interpretation the question of simultaneous definability of observables corresponding to non-commuting operators is irrelevant, since the Copenhagen interpretation deals exclusively with measurement outcomes.

From the above analysis, it is clear that the quantities with the $\Delta$ in equation (3.9) refer to the statistical properties of the coherent states, while $\delta p$ and $\delta q$ refer to the difference between $|z\rangle$ and $|z+\delta z\rangle$. If we interpret the decoherence functional in terms of measurements this is the difference between two filters measuring the phase space properties of the physical system.

No physical measurement device has infinite accuracy on phase space, because the Heisenberg uncertainty relation sets a bound on the sharpness we can achieve by means of phase space measurements. Hence,

$$
\delta q \delta p \sim 1
$$

Taking this into account, the infinitesimal distance $\delta s^{2}$ satisfies

$$
\delta s^{2} \geq(\Delta p)^{2} \delta q^{2}+\frac{(\Delta q)^{2}}{\delta q^{2}}+2 C_{p q}
$$

Minimising the right-hand-side with respect to $\delta q$ we obtain

$$
\delta s^{2} \geq 2\left(\Delta p \Delta q+C_{p q}\right)
$$

We can further simplify this expression by using a generalised statistical uncertainty relation [33]

$$
(\Delta q)^{2}(\Delta p)^{2}-C_{p q}^{2} \geq \frac{1}{4}
$$

which implies that

$$
\left|C_{p q}\right| \leq \sqrt{(\Delta q)^{2}(\Delta p)^{2}-\frac{1}{4}}
$$

So we have

$$
\begin{array}{r}
\Delta p \Delta q+C_{p q} \geq \Delta p \Delta q-\left|C_{p q}\right| \\
\geq \Delta p \Delta q-\sqrt{(\Delta q)^{2}(\Delta p)^{2}-\frac{1}{4}} \geq \frac{1}{2} .
\end{array}
$$

Substituting into (3.12) we get

$$
\delta s^{2} \geq 1
$$

This inequality is important, because it defines the operational limit in taking continuous phase space measurements using coherent states. The change $\delta z$ in the argument of the coherent state cannot be made that small (even if it is only in the $q$ direction) as to make meaningful the procedure of taking the continuum limit. There exists a minimum length on phase space given by $2 \Delta p \Delta q+C_{p q}$ and 
any phase space process we monitor consists of discrete steps of this order of magnitude. This minimum length depends on the choice of coherent states, in other words on the choice of the filters we use in order to describe phase space properties. Irrespective of the choice made the precision cannot be rendered smaller than one.

We note here that we can also minimise the phase space distance with respect to all possible choices of coherent states. For this purpose we use equation (3.13) to get

$$
\begin{gathered}
\delta s^{2} \geq(\Delta p)^{2} \delta q^{2}+(\Delta q)^{2} \delta p^{2}-2\left|C_{p q}\right| \delta p \delta q \geq \\
(\Delta p)^{2} \delta q^{2}+(\Delta q)^{2} \delta p^{2}-\sqrt{(\Delta q)^{2}(\Delta p)^{2}-\frac{1}{4}}
\end{gathered}
$$

The right-hand side has a maximum, when the term in the square root vanishes. In this case,

$$
\delta s^{2} \geq \frac{\delta q^{2}}{4(\Delta q)^{2}}+(\Delta q)^{2} \delta p^{2} \geq \delta q \delta p
$$

This result makes it clear that the relation $\delta s^{2} \geq 1$ is equivalent to Heisenberg's uncertainty relation. Moreover since $\delta s^{2}$ has a lower bound, the corresponding term in the decoherence functional cannot be made to vanish, at least for the case of measurements. Hence, the quantum anti-Zeno effect (or the quantum Zeno effect) cannot really arise in a concrete measurement situation; see also a similar analysis in [34].

\subsubsection{Similarity to a diffusion process}

In taking the continuum limit, we have assumed that the paths $z(\cdot)$ are continuous functions of a time parameter $t$. In standard treatments this parameter is identified with Newtonian time. In that case $\delta z \sim \delta t$ and $\delta s^{2} \sim \delta t^{2}$ and the term with the metric in the decoherence functional (3.8) goes to zero.

However, in equation (3.8) the specification of the time parameter appears nowhere as we assumed a vanishing Hamiltonian. The decoherence functional and probabilities are written solely in terms of the $\delta z$. But this does not mean that the notion of time is lost. Recall the distinction of the two properties of time: its function as a causal ordering parameter and its function as the parameter measuring evolution are distinct [10]. Even with vanishing Hamiltonian the causal ordering is still present: it is the sequence by which the various single time properties $z_{i}$ enter in order to form a path. The ordering structure does not depend on the numerical value of the time parameter; it suffices to designate which property is first (or if one prefers which measurement takes place first.) There is no a priori reason to consider external Newtonian time as the parameter determining the path. 
More precisely, the uncertainty relation $\delta s^{2} \geq 1$ strongly suggests that the paths $z(\cdot)$ cannot be taken as continuous (or at least differentiable) functions of the time $t$, for in this case $\delta s^{2}$ could be made arbitrarily small by taking $\delta t$ going to zero.

Let us consider now an (N+1)-time fine-grained history $\alpha=\left(z_{0}, z_{1}, z_{2}, \ldots z_{N}\right)$. If we write $\delta z_{i}=z_{i}-z_{i-1}$, the probability for $\alpha$ is

$$
p(\alpha)=e^{-\sum_{i} \delta s_{i}^{2}} \geq e^{-\sum_{i}^{1}}=e^{-N},
$$

where the inequality is due to the uncertainty relation. This seems like a nice behaviour: the more single-time measurements involved in the monitoring of a path, the smaller the probability that we are going to get it right.

On the other hand, the behaviour of the limiting probability for a path going with $e^{-N}$ is similar to that of classical decay process. We must note here that some caution is needed in the drawing of any quantitative conclusions from such analogies, since the quantum probabilities (3.19) do not correspond to an additive probability measure. Nonetheless, the analogy helps to demonstrate some interesting points.

Let us consider a Wiener process on a Riemannian manifold. This is associated to the heat equation for the single-time probability distribution

$$
\frac{\partial}{\partial t} \rho=D \nabla^{2} \rho
$$

with $D$ a diffusion constant. The probability for a small transition

$$
p(\alpha)=e^{-\frac{D}{2 \delta t} \delta s^{2}} .
$$

Unlike (3.9) this expression is exact for all values of $\delta t$ and $\delta z$.

In a Wiener process the time $t$ appears explicitly. Then one can define the derivative $\dot{z}$ with respect to $t$ and write the probability distribution for a path

$$
p(z(\cdot)) \sim e^{-\frac{D}{2} \int d t g_{i j}(z) \dot{z}^{i} \dot{z}^{j}} .
$$

This expression is formal: one needs to specify the discrete version of the integral, because most paths in which the measure is defined are not differentiable ${ }^{6}$. However, these issues can be ignored since we are only interested in the formal comparison of (3.24) with the quantum mechanical equation (3.9)

Clearly the main difference between the two equations is the presence of the term $\delta t$ in the denominator of the exponent of the probability. This signifies the appearance of Newtonian time in the probability measure and guarantees its finiteness.

To follow this analogy in the quantum case we introduce a time-step $1 / \nu$, which corresponds to the duration of a single time-step in terms of Newtonian

\footnotetext{
${ }^{6}$ This is in analogy with stochastic processes and it is the reason the terms with the metric, which are of order $(\delta z)^{2}$ cannot be ignored in the continuum limit.
} 
time. For timescales much larger than $\nu^{-1}$ we can then approximate $z(t)$ by a continuous path. In this case $\delta z^{i}=\frac{1}{\nu} \dot{z}^{i}$ and the probability reads

$$
p(z(\cdot)) \sim e^{-\frac{1}{\nu} \int_{0}^{t} d s g_{i j}(z) \dot{z}^{\dot{z}} \dot{z}^{j}} .
$$

Comparing with (3.13) we see that $\nu^{-1}$ is analogous to a diffusion coefficient.

In the same approximation the decoherence functional (3.8) reads

$$
d(\alpha, \beta)=\exp \left(i \int_{C} A-\frac{1}{2 \nu} \int_{C} d s g_{i j}(z) \dot{z}^{i} \dot{z}^{j}\right) .
$$

This expression converges to the Berry phase for $\nu \rightarrow \infty$. In order to avoid any misunderstanding, we should remark that the expression (3.24) for the decoherence functional is completely different and leads to different properties from the ones arising in studies of decoherence in open quantum systems.

The parameter $\nu$ was first introduced by Klauder as a means of regularising the coherent state path integral in a geometrical fashion [36]. He introduced $\nu$ as a diffusion coefficient of a Wiener process on phase space, which is eventually taken to infinity. He proved that the coherent state propagator equals

$$
\left\langle z\left|e^{-i \hat{H} t}\right| z^{\prime}\right\rangle=\lim _{\nu \rightarrow \infty} \int D z(\cdot) e^{\nu t} e^{i \int A-i \int_{0}^{t} d s H-\frac{1}{2 \nu} \int_{0}^{t} d s g_{i j} \dot{z}^{i} \dot{z}^{j}} .
$$

However, if $\nu$ is not taken to infinity, the propagation is not unitary. Klauder and Maraner explored this case and they showed that in the deterministic regime characterised by the saddle-point approximation of (3.27) many features of quantum theory can be recovered [37]. They commented, however, that if one takes this picture seriously, one would have to deal with the constraints of Bell's theorem. However, this is not problematic when one does not seek a deterministic theory on phase space: the description by means of the decoherence functional employs non-additive probabilities and is, therefore, not constrained by Bell's theorem.

\subsection{Introducing dynamics: time parameterisation}

\subsubsection{The extended phase space}

In our work so far, the external Newtonian time did not appear explicitly in the decoherence functional. This was due to the absence of the Hamiltonian, which is the generator of translations in Newtonian time.

If we take the Hamiltonian operator into account the kernel $\left\langle z\left|e^{-i \hat{H}\left(t-t^{\prime}\right)}\right| z^{\prime}\right\rangle$ appears in the expression (2.7) for the decoherence functional. It is convenient to define the new coherent states

$$
|z, t\rangle=e^{-i \hat{H} t}|z\rangle
$$


Here the vector $|z, t\rangle$ is parameterised by elements of $\Gamma \times \mathbf{R}$. The vectors $|z\rangle$ will still form coherent states in the generalised sense, as they provide a map from $\Gamma \times \mathbf{R}$ to the projective Hilbert space. In this case there does not exist a decomposition of the unity, but one can still pullback the metric and connection to $\Gamma \times \mathbf{R}$.

In what follows we shall write $Z=(z, t)$ and use Greek letters for the indices of the tensors on $\Gamma \times \mathbf{R}$, so that $Z^{0}:=t$ and $Z^{i}:=z^{i}$. It is convenient to define the operators $\hat{A}^{\mu}$. Explicitly

$$
\hat{A}_{0}=\hat{H},
$$

and $\hat{A}_{i}$ is defined by its action on coherent states

$$
\hat{A}_{i}|z\rangle=-i\left|\partial_{i} z\right\rangle .
$$

We denote tensors on $\Gamma \times \mathbf{R}$ with an overbar. In this notation

$$
\begin{array}{r}
\bar{A}_{\mu}(Z)=\left\langle Z\left|\hat{A}_{\mu}\right| Z\right\rangle \\
\bar{g}_{\mu \nu}=\left\langle Z\left|\hat{A}_{\mu} \hat{A}_{\nu}\right| \bar{Z}\right\rangle-\left\langle Z\left|\hat{A}_{\mu}\right| Z\right\rangle\left\langle Z\left|\hat{A}_{\nu}\right| Z\right\rangle
\end{array}
$$

On $\Gamma \times \mathbf{R}$ the two form

$$
\bar{\Omega}=d \bar{A}=\Omega-d H(z) \wedge d t
$$

is degenerate. This means that $\Gamma \times \mathbf{R}$ is a presymplectic manifold and $\Gamma$ is obtained by excising the degenerate directions of $\Omega$.

Alternatively, this process can be described in terms of the theory of constraints. To see this consider the manifold $\Gamma \times \mathbf{R}^{2}$ with points $\left(z, t, p_{t}\right)$. The two-form $\Omega+d p_{t} \wedge d t$ is non-degenerate, thus $\Gamma \times \mathbf{R}^{2}$ is a symplectic manifold. If we consider the first-class constraint

$$
\phi\left(z, t, p_{t}\right)=p_{t}+H(z)=0,
$$

then $\Gamma \times \mathbf{R}$ together with the two-form (3.30) is the constraint surface. We can obtain $\Gamma$ by the standard procedure of symplectic reduction, i.e. $\Gamma$ is defined as the set of all orbits on the constraint surface under the symplectic transformations generated by the constraint. First-class constrained systems with this behaviour are called parameterised and have many common features with general relativity.

Now, we can write the decoherence functional in complete analogy to (.14)

$$
d(\alpha, \beta)=\exp \left(i \sum_{a} \bar{A}\left(Z_{a}+\frac{\delta Z_{a}}{2}\right) \delta z_{a}-\frac{1}{2} \sum_{a} \delta \bar{s}_{a}^{2}\right)+O\left(\delta z^{3}\right) .
$$

If we consider continuous paths we obtain

$$
d(\alpha, \beta)=e^{i \int_{C} \bar{A}}=e^{i \int_{C}(A-H d t)}=e^{i S},
$$


which means that the decoherence functional equals the value of the phase space action along the closed loop formed by the two histories.

We should present here an important point of the history formalism. While we could in principle study any path on $\Gamma \times \mathbf{R}$, physical histories are only the ones for which the time parameter increases along the physical temporal ordering. This means that if we have a history $\alpha=\left(Z_{1}, Z_{2}, \ldots, Z_{n}\right)$ it cannot be physical unless $t\left(Z_{1}\right)<t\left(Z_{2}\right)<\ldots t\left(Z_{n}\right)$. If this is not the case then any predictions will be nonsensical as the time ordering enters crucially in the definition of the decoherence functional. The exact values of the time parameter do not matter as long as the ordering is preserved. This is another reflection of the distinction between the ordering and the evolutionary properties of time, which characterises any history description [10]. For example, if we work on a parameterised system we need to specify a time-ordering in the space of all possible histories [41, 42]. In the above case time is assumed Newtonian and the ordering is trivial; it could be more interesting in a consideration of relativistic systems.

\subsubsection{Time-energy uncertainty relation}

Once more we engage in the discussion of the uncertainty relation. The metric on the extended phase space reads

$$
\delta \bar{s}^{2}=\delta s^{2}(t)+2 C_{E A_{i}} \delta z^{i} \delta t+(\Delta E)^{2} \delta t^{2} .
$$

Here $C_{E A_{i}}$ is the correlation between the Hamiltonian and the operator $\hat{A}_{i}(t)=$ $e^{i \hat{H} t} \hat{A}_{i} e^{-\hat{H} t}$, i.e.

$$
C_{E A_{i}}=\frac{1}{2}\left\langle z\left|\hat{A}_{i}(t) \hat{H}+\hat{H} \hat{A}_{i}(t)\right| z\right\rangle-\left\langle Z\left|\hat{A}_{i}(t)\right| Z\right\rangle\langle Z|\hat{H}| Z\rangle,
$$

while $(\Delta E)^{2}$ is the energy uncertainty on the coherent state $|z\rangle^{7}$.

In order to simplify the expressions, we define the operators

$$
\begin{array}{r}
\hat{C}=\Delta \hat{A}_{i}(t) \delta z^{i}=\hat{A}_{i}(t) \delta z^{i}-\left\langle Z\left|\hat{A}_{i}(t)\right| Z\right\rangle \delta z^{i} \\
\hat{D}=-\Delta \hat{H} \delta t=-(\hat{H} \delta t-\langle Z|\hat{H}| Z\rangle \delta t) .
\end{array}
$$

We then write the following inequality

$$
\left\langle\psi\left|\hat{C}^{2}\right| \psi\right\rangle\left\langle\psi\left|\hat{D}^{2}\right| \psi\right\rangle-\frac{1}{4}(\langle\psi|\hat{C} \hat{D}+\hat{D} \hat{C}| \psi\rangle)^{2} \geq \frac{1}{4}|\langle\psi|[\hat{C}, \hat{D}]| \psi\rangle|^{2},
$$

for which inequality $(3.15)$ is a special case ${ }^{8}$. Now

$$
[\hat{C}, \hat{D}]=-\left[\hat{A}_{i}(t), \hat{H}\right] \delta z^{i} \delta t=-e^{i \hat{H} t}\left[\hat{A}_{i} \delta z^{i}, \hat{H}\right] e^{-i \hat{H} t} \delta t .
$$

\footnotetext{
${ }^{7}$ Note that $\delta \bar{s}^{2}(t)=(\Delta \delta \hat{S})$, where $\delta \hat{S}=\hat{A}_{i}(t) \delta z^{i}-\hat{H} \delta t$ is the operator that measures the change of action corresponding to the the transition of $\delta z^{i}$ and $\delta t$.

${ }^{8}$ Explicit writing down of all terms in (3.41) will convince the reader that this is a consequence of Schwartz's inequality.
} 
The commutator equals $\frac{\partial}{\partial z^{i}} \hat{H} \delta z^{i}$, which in turn equals $\delta \hat{H}$, i.e. the change in the Hamiltonian by virtue of a translation by $\delta z^{i}$ on phase space.

If we define

$$
\delta H(z, t)=\left\langle z\left|e^{i \hat{H} t} \delta \hat{H} e^{-i \hat{H} t}\right| z\right\rangle
$$

we obtain

$$
[\hat{C}, \hat{D}]=-\delta H(t, z) \delta t .
$$

The inequality (3.41) implies that (after we drop $\psi$ for simplicity)

$$
|\langle\hat{C} \hat{D}+\hat{D} \hat{C}\rangle| \leq \sqrt{4\left\langle C^{2}\right\rangle\left\langle D^{2}\right\rangle-\delta H^{2} \delta t^{2}},
$$

hence,

$$
\delta \bar{s}^{2} \geq\left\langle\hat{C}^{2}\right\rangle+\left\langle\hat{D}^{2}\right\rangle-\sqrt{4\left\langle C^{2}\right\rangle\left\langle D^{2}\right\rangle-\delta H^{2} \delta t^{2}} .
$$

Now the right-hand-side takes maximum value when the term in the square root vanishes,

$$
4\left\langle C^{2}\right\rangle\left\langle D^{2}\right\rangle=\delta H^{2} \delta t^{2}
$$

Then

$$
\delta \bar{s}^{2} \geq\left\langle\hat{C}^{2}\right\rangle+\frac{\delta H^{2} \delta t^{2}}{4\left\langle C^{2}\right\rangle} .
$$

We can minimise the right-hand-side of (3.48) with respect to $\left\langle C^{2}\right\rangle$ to get

$$
\delta \bar{s}^{2} \geq \delta H \delta t
$$

where $\delta H$ is the difference in the classical value of the energy between two specifications of phase space points.

Due to the uncertainty in the specification of phase space points there exists an uncertainty $\delta E$ in the specification of the energy: $\delta H$ cannot be smaller than this energy. Taking into account the time-energy uncertainty principle for $\delta E$ ${ }^{9}$, namely $\delta E \delta t \sim 1$, we get

$$
\delta \bar{s}^{2} \geq 1
$$

So far we have seen that the quantum evolution of a quantum system with non-zero Hamiltonian is best described in terms of the geometry of the extended phase space, which includes time as a parameter. The condition for the Riemannian metric on $\Gamma_{e x t}, \delta \bar{s}^{2} \sim 1$, is equivalent to Heisenberg 's uncertainty relation. Note that the derivation of this equation did not specify the physical system's phase space. Hence we conclude that the geometric description can be used to implement Heisenberg's principle in any physical system, including systems that are described by topologically non-trivial phase spaces.

\footnotetext{
${ }^{9}$ The time-energy uncertainty relation exists only in one form, the Heisenberg one. There does not exist a statistical form, because quantum theory does not accept a physical time operator. See [2] for a recent review.
} 
Like in the case of vanishing Hamiltonian, for a history $\alpha$ with $\mathrm{N}$ time-steps the probability is

$$
p(\alpha) \sim e^{-N} .
$$

We can write a sharper inequality for this probability. If we assume that $\delta H(t)>\delta E_{t}$, we can go to the continuous limit, namely

$$
p(\alpha) \sim e^{-\int d t \delta E_{t}},
$$

It is important to remark that $\delta E_{t}$ is not proportional to $\delta t$ as it corresponds to an irreducible spread of energy of the quantum state at a moment of time. Equation (3.52) is very interesting. It strongly suggests that the assignment of probabilities to histories primarily depends on the time-averaged energy uncertainty $\langle\delta E\rangle=\frac{1}{t} \int_{0}^{t} d s \delta E_{s} d s$ for the paths: the most probable paths being characterised by the smallest values of $\langle\delta E\rangle$. Essentially $(\langle\delta E\rangle)^{-1}$ is the decay time of the probabilities for the continuous paths. The metric also contributes to the off-diagonal elements of the decoherence functional. Paths characterised by large values of time-averaged energy uncertainty seem to decohere more efficiently.

\section{An example: spinless relativistic particle}

\subsection{The coherent states}

As a non-trivial application of our previous ideas we study the phase space geometry of a relativistic particle with zero spin. The relevant symmetry group is the Poincaré group, so we construct its associated coherent states.

We recall here that the Poincaré group is the semidirect product of the Lorentz group with the Abelian group of spacetime translations. Its representations are characterised by the value of the mass $m$ and spin $s$. In this paper we shall study the case $m \neq 0, s=0$, namely a massive spinless particle.

We consider the space of all unit, timelike vectors $\xi$, with positive values of the zero-th component, $V=\left\{\xi \mid \xi_{\mu} \xi^{\mu}=1, \xi^{0} \geq 0\right\}$. The space $V$ carries a Poincaré invariant measure

$$
d \mu(\xi)=m^{2} \frac{d^{3} \xi}{2 \omega_{\xi}}
$$

where $\omega_{\xi}=\xi^{0}=\sqrt{1+\xi^{2}}$.

The Hilbert space on which the representation is constructed is $\mathcal{L}^{2}(V, d \mu)$ 
and the Poincaré group action ${ }^{10}$

$$
\begin{array}{r}
\hat{U}(\Lambda) \Psi(\xi)=\Psi\left(\Lambda^{-1} \xi\right), \\
\hat{U}(X) \Psi(\xi)=e^{i m \xi \cdot X} \Psi(\xi) .
\end{array}
$$

In order to construct a set of coherent states we need to choose a reference vector $\Psi_{0}$, which is invariant under the action of the maximal compact subgroup of the Poincaré group, namely the group $S O(3)$ of spatial rotations. This suggests that the reference vector depends on $\xi$ only through the product $n_{\mu} \xi^{\mu}$, where $n_{\mu}$ is a unit timelike vector.

We choose a Gaussian reference vector

$$
\Psi_{0}(\xi)=\frac{1}{m\left(\pi \sigma^{2}\right)^{3 / 2}}(2 n \cdot \xi)^{1 / 2} e^{-\frac{1}{2 \sigma^{2}} \xi^{n} \Gamma \cdot \xi}
$$

where ${ }^{n} \Gamma_{\mu \nu}=-\eta_{\mu \nu}+n_{\mu} n_{\nu}$. This vector is centered around $\xi^{i}=0$ with a width equal to $\sigma$.

For fixed $n$ we can distinguish the elements of the Lorentz group into the ones that leave $n$ invariant (which form a subgroup $\mathrm{SO}(3)$ of spatial rotations) and the ones that do not. The latter generate boosts and can be parameterised by a unit timelike vector $I$, such that

$$
\Lambda_{I} n=I .
$$

The above equation defines an isomorphism, because for each $I$ there exists a unique $\Lambda_{I}$ satisfying (4.9) and vice versa. Any Lorentz matrix can be written as a product $\Lambda=R \Lambda_{I}$ for some rotation matrix and vector $I$. When we act on our reference vector with $\Lambda$, the rotation matrix does not contribute as it leaves the product $n \cdot \xi$ invariant. Hence only the boost part of the Lorentz matrix acts non-trivially. Thus the coherent states associated to the Poincaré group depend on $I$ and the parameters $X$, which correspond to spacetime translations,

$$
\begin{array}{r}
\Psi_{X, I}(\xi)=\hat{U}(X) \hat{U}\left(\Lambda_{I}\right) \Psi_{0}(X)=e^{i m \xi \cdot X} \Psi_{0}\left(\Lambda_{I}^{-1} \xi\right)= \\
\frac{1}{m\left(\pi \sigma^{2}\right)^{3 / 2}}(2 I \cdot \xi)^{1 / 2} \exp \left(-\frac{1}{2 \sigma^{2}}\left(I_{\mu} I_{\nu}-\eta_{\mu \nu}\right) \xi^{\mu} \xi^{\nu}+i m \xi_{\mu} X^{\mu}\right) .
\end{array}
$$

An interesting point is to examine the expectation value of the energymomentum vector on the coherent states

$$
\left\langle X, I\left|\hat{P}^{\mu}\right| X, I\right\rangle=m \kappa I^{\mu},
$$

\footnotetext{
${ }^{10}$ Let us denote by $\hat{P}^{\mu}$ and $\hat{M}^{\mu \nu}$ the generators of the translations and the Lorentz group respectively. We can distinguish the boost generators $\hat{K}^{i}=\hat{M}^{0 i}$ from the rotation ones $\hat{N}^{i}=\frac{1}{2} \epsilon^{i j k} \hat{M}_{i j}$, by making reference to a timelike direction. The generators read explicitly $\hat{P}^{\mu}=\xi^{\mu}, \hat{\mathbf{N}}=-i \xi \times \nabla_{\xi}$ and $\hat{\mathbf{K}}=-i \xi^{0} \nabla_{\xi}$, where $\left(\nabla_{\xi}\right)_{i}=\frac{\partial}{\partial \xi^{i}}+\frac{\xi^{i}}{\xi^{0}} \frac{\partial}{\partial \xi^{0}}$.
} 
where

$$
\kappa=\int d \mu(\xi) \omega_{\xi}\left|\Psi_{0}\right|^{2}(\xi)
$$

is the expectation value of the energy $n_{\mu} \hat{P}^{\mu}$ on $\Psi_{0}$ divided by $m$. Because the coherent state is not localised at a point of $V$ there exists a spread in the distribution of energy, even though the spatial momenta vanish. If equation (4.7) is to conform with our expectations that the expectation value of $\hat{P}^{\mu}$ is the momentum associated to the coherent states, we need to redefine the mass on phase space as $M=\kappa m$. We shall later show that this is the correct account.

We can estimate $\kappa$

$$
\kappa=1+\frac{1}{4} \sigma^{2}-\frac{1}{16} \sigma^{4}+O\left(\sigma^{6}\right)
$$

The set of coherent states (4.6) depends on seven parameters, three corresponding to the momenta $I$ and four corresponding to the spacetime translations. Clearly one of these parameters plays the role of time, hence these coherent states are similar to the states $|z, t\rangle$ of the previous section. They are not expected to have a decomposition of the unity.

Furthermore these states do not depend on the choice of $n$, even though the reference vector did. The set of coherent states is invariant under the action of the Poincaré group

$$
\begin{gathered}
\hat{U}(\Lambda)|X, I\rangle=|\Lambda X, \Lambda I\rangle \\
\hat{U}(Y)|X, I\rangle=|X+Y, I\rangle .
\end{gathered}
$$

We can calculate the fine-grained decoherence functional (3.35) in terms of these coherent states. Note again that in the specification of any path the causality condition has to be specified. Hence a phase space point $\left(X^{\prime}, I^{\prime}\right)$ is in the future of another phase space point $(X, I)$ only if the causal structure of Minkowski spacetime is respected. That is, $X^{\prime}$ has to lie in the causal future of $X$. This means that $X$ and $X^{\prime}$ have to be timelike or null separated and $X^{\prime 0} \geq X^{0}$. Furthermore, in order to describe physical particles we demand that $I^{0}>0$.

We can reduce the set of coherent states by taking a fixed value of the parameter $t=n \cdot X$, i.e. treating $t$ as an external parameter and not as an argument of the coherent states.

In this way we may define our coherent states at an instant of time, a spacelike three-surface $\Sigma$, which is uniquely determined by the choices of $n$ and $t$. The coherent states then depend on the spatial variables $x$ and $I$, which are the projections of $X$ and $I$ on $\Sigma$ and they span the phase space of a single particle $T^{*} \Sigma$.

We denote the coherent states restricted on $\Sigma$ as $|x, I\rangle_{\Sigma}$. The Poincaré group behaves as follows: transformations that leave $\Sigma$ invariant (spatial rotations and translations) preserve the coherent states, while the ones that take $\Sigma$ to another 
surface $\Sigma^{\prime}$ (namely boosts and time translations) also take the set of coherent states into the one associated to $\Sigma^{\prime}$.

For the restricted coherent states we can calculate

$$
\left.\int d^{3} I d^{3} x\langle\xi \mid x, I\rangle_{\Sigma \Sigma}\right\rangle x, I\left|\xi^{\prime}\right|=\frac{1}{m^{3}} 2 \kappa \omega_{\xi} \delta^{3}\left(\xi-\xi^{\prime}\right),
$$

and thus implies the existence of a resolution of the unity

$$
\kappa \hat{1}=m^{3} \int d^{3} I d^{3} x|x, I\rangle_{\Sigma \Sigma}\langle x, I|
$$

In what follows, we shall denote the measure $m^{3} d^{3} I d^{3} x$ as $d \mu_{\Sigma}(I, x)$.

Since the decomposition of the unity is necessary in order to define the operators that represent physical quantities, any such definition necessarily depends on the choice of the surface $\Sigma$. For example, this is the case of the position operator

$$
\hat{\mathbf{x}}_{\Sigma}=\frac{1}{\kappa} \int d \mu_{\Sigma}(I, x) \mathbf{x}|x, I\rangle_{\Sigma \Sigma}\langle x, I|,
$$

which coincides with the one defined by Newton and Wigner [43].

Hence, even if the fine-grained decoherence functional is a fully covariant object, the specification of any correlation function makes reference to some spacelike surface $\Sigma$ and hence breaks the covariance. This is also true for the coarse-grained histories. Coarse-graining over phase space involves integration with the measure $d \mu_{\Sigma}$ and hence refers to a spacelike surface. Hence any prediction of the theory carries implicitly a reference to a chosen hypersurface. This is already known in canonical quantisation and is highlighted in the histories formalism [40].

\subsection{Phase space geometry}

The first step towards determining the geometric objects on $\Gamma$ is to compute $d|X, I\rangle$. It equals

$$
d \Psi_{I X}(\xi)=\left[\frac{\xi \cdot d I}{2 I \cdot \xi}-\frac{\xi \cdot I \xi \cdot d I}{\sigma^{2}}+i m \xi \cdot d X\right] \Psi_{I X}(\xi)
$$

from which we obtain

$$
\langle X, I|d| X, I\rangle=i m \kappa I^{\mu} d X_{\mu}+\frac{1}{2}\left(1-\frac{\kappa}{\sigma^{2}}\right) I^{\mu} d I_{\mu} .
$$

The fact that $I^{2}=1$ implies that $I_{\mu} d I^{\mu}=0$, hence

$$
\begin{array}{r}
A=\kappa m I^{\mu} d X_{\mu}, \\
\Omega=\kappa m d I^{\mu} \wedge d X_{\mu} .
\end{array}
$$


It is clear from the above that the correct definition of the classical momentum is $P^{\mu}=m \kappa I^{\mu}$. However, this also implies that $P_{\mu} P^{\mu}=\kappa^{2} m^{2}{ }^{11}$.

The calculation of the metric is straightforward but tedious. The end result is

$$
d s^{2}=-\frac{\alpha}{3 \sigma^{2}} \eta_{\mu \nu} d I^{\mu} d I^{\nu}+K_{\mu \nu} d X^{\mu} d X^{\nu} .
$$

The first term is the Riemannian metric on $V$ inherited from the Lorentzian metric on Minkowski spacetime times a constant. The parameter $\alpha$ equals

$$
\alpha=\frac{1}{\left(\pi \sigma^{2}\right)^{1 / 2}} \int_{0}^{\infty} \frac{d \xi}{1+\xi^{2}} e^{-\xi^{2} / \sigma^{2}}=1+O\left(\sigma^{2}\right) .
$$

The second term is characterised by the tensor

$$
K^{\mu \nu}=\left\langle X, I\left|\hat{P}^{\mu} P^{\nu}\right| X, I\right\rangle-\left\langle X, I\left|\hat{P}^{\mu}\right| X, I\right\rangle\left\langle X, I\left|\hat{P}^{\nu}\right| X, I\right\rangle,
$$

which is the correlation tensor for the four-momentum on a coherent state. Explicitly,

$$
K_{\mu \nu}=m^{2}\left[\left(1+\frac{2}{3} \sigma^{2}-\kappa^{2}\right) I_{\mu} I_{\nu}-\frac{1}{6} \sigma^{2} \eta_{\mu \nu}\right] .
$$

We want the coherent state to be very sharply peaked around $\xi^{\mu}=I^{\mu}$, so that it will correspond as closely as possible to a phase space point. For this purpose we take $\sigma<<1$. In this case the dominant terms are

$$
\delta \tilde{s}^{2}=\left(\frac{1}{3 \sigma^{2}}+O\left(\sigma^{0}\right)\right) \eta_{\mu \nu} \delta I^{\mu} \delta I^{\nu}+m^{2}\left(\frac{\sigma^{2}}{6}+O\left(\sigma^{4}\right)\right)\left(I_{\mu} I_{\nu}-\eta_{\mu \nu}\right) \delta X^{\mu} \delta X^{\nu} .
$$

If we minimise the right-hand-side with respect to $\sigma^{2}$ we get

$$
\delta \tilde{s}^{2} \geq m \frac{\sqrt{2}}{3} \delta I \delta_{I} X
$$

where

$$
\begin{aligned}
\delta I=\sqrt{-\eta_{\mu \nu} \delta I^{\mu} \delta I^{\nu}} & =\sqrt{\left(\delta_{i j}-\frac{I_{i} I_{j}}{1+I^{2}}\right) \delta I^{i} \delta I^{j}} \\
\delta_{I} X & =\sqrt{\left(I_{\mu} I_{\nu}-\eta_{\mu \nu}\right) \delta X^{\mu} \delta X^{\nu}} .
\end{aligned}
$$

\footnotetext{
${ }^{11}$ In quantum theory the Wigner representation theory says that the spinless representations of the Poincaré group are classified by the value of the parameter $m=\hat{P}^{\mu} \hat{P}_{\mu}$.In symplectic mechanics the theory of Konstant-Souriau states that the symplectic actions of the Poincaré group (in the spinless case) is classified by the value of $P_{\mu} P^{\mu}=M^{2}$. The coherent states provide a map between quantum theories corresponding to parameter $m$ and classical symplectic manifolds characterised by the value $M$. There is no a priori reason why these parameters should have the same value.
} 
The uncertainty relation $\delta \tilde{s}^{2} \sim 1$ implies that

$$
m \delta I \delta_{I} X \geq 1 .
$$

Equation (4.24) provides a covariant generalisation of the time-energy uncertainty relation.

In the particle's rest frame we have $I^{\mu}=n^{\mu}$. Therefore the condition $I^{\mu} \delta I_{\mu}=0$ implies that $\delta I^{0}=0$, so that

$$
\begin{aligned}
\delta I & =\sqrt{\delta \mathbf{I} \cdot \delta \mathbf{I}}, \\
\delta_{I} X & =\sqrt{\delta \mathbf{x} \cdot \delta \mathbf{x}},
\end{aligned}
$$

which means that (4.31) coincides with the non-relativistic uncertainty relation.

Of special interest is the degenerate case that $\delta X^{\mu}=\delta t I^{\mu}$. This corresponds to the case that the measuring devices are clocks along the classical trajectory of the particle. In this case the dominant term of $K_{\mu \nu}$ vanishes and we have to use the next term in the expansion of $\kappa$. This yields

$$
\delta \bar{s}^{2}=\frac{m^{2}}{3 \sigma^{2}}(\delta I)^{2}+\frac{m^{2} \sigma^{4}}{16}(\delta t)^{2} .
$$

Minimising with respect to $\sigma$ we get

$$
\delta \bar{s}^{2} \geq \frac{3^{1 / 3}}{4} m^{2 / 3}(\delta I)^{4 / 3}(\delta t)^{2 / 3} .
$$

This implies that the Heisenberg uncertainty relations take a rather unusual form

$$
m^{2 / 3}(\delta t)^{2 / 3}(\delta I)^{4 / 3} \sim 1 .
$$

\section{Conclusions}

We have studied the role that the phase space geometry plays in the probability assignment for continuous-time histories. In particular, we showed that the coherent states induce a metric on the phase space, which proves to be a key ingredient of the decoherence functional.

This metric has a significant physical interpretation as it provides a geometric way of formulating the Heisenberg uncertainty relations. This version of the uncertainty relations can be implemented in any classical phase space, including the ones with non-trivial topological structure. An interesting byproduct of our results is a new proof of the impossibility of the physical manifestation of the quantum Zeno and anti-Zeno paradoxes.

It is important to remark that in the generic case, that the Hamiltonian does not vanish, the physically relevant metric is defined on the extended phase space, which includes time as a parameter. Since the decoherence functional 
is constructed from the metric and the phase space action, our construction provides a stepping stone for a geometric quantisation algorithm for histories, which can be naturally generalised to include parameterised systems, such as the relativistic particle we considered in section 5. For this reason it might prove relevant in the histories quantisation of general relativity [44]. In fact, the search for a geometric procedure for the quantisation of histories has been one of the main motivations of this work.

Finally, we note that our construction of relativistic coherent states and the ensuing uncertainty relations are novel objects, which demonstrate the versatility of our approach for the study of the quantum properties of a large class of physical systems.

\section{Acknowledgements}

We gratefully acknowledge support from the EPSRC GR/R36572 grant (N.S.) and from the Marie Curie Fellowship of the European Commission (C.A).

\section{References}

[1] W. Heisenberg, Über den anschaulichen Inhalt der quantentheoretischen Kinematik und Mechanik, Z. Physik 69, 56 (1927).

[2] P. Busch, The Time-Energy Uncertainty Relation, quant-ph/0105049.

[3] W. Unruh and R. Wald, Time and the Interpretation of Quantum Gravity, Phys. Rev D40, 2598 (1989).

[4] R. B. Griffiths. Consistent Histories and the Interpretation of Quantum Mechanics, J. Stat. Phys. 36, 219, 1984.

[5] R. Omnès, Logical Reformulation of Quantum Mechanics: I Foundations, J. Stat. Phys. 53, 893, 1988; The Interpretation of Quantum Mechanics, (Princeton University Press, Princeton, 1994); Consistent Interpretations of Quantum Mechanics, Rev. Mod. Phys. 64, 339, 1992.

[6] M. Gell-Mann and J. B. Hartle, Quantum mechanics in the Light of Quantum Cosmology, in Complexity, Entropy and the Physics of Information, edited by W. Zurek, (Addison Wesley, Reading, 1990); Classical Equations for Quantum Systems, Phys. Rev. D 47, 3345, 1993.

[7] J. B. Hartle, Spacetime Quantum Mechanics and the Quantum Mechanics of Spacetime, in Proceedings on the 1992 Les Houches School, Gravitation and Quantisation, 1993.

[8] C. J. Isham, Quantum Logic and the Histories Approach to Quantum Theory, J. Math. Phys. 35, 2157, 1994. 
[9] B. Misra and E. C. G. Sudarshan, The Zeno's Paradox in Quantum Theory, J. Math. Phys. 18, 657 (1977); C. B. Chiu, B. Misra and E. C. G. Sudarshan, Time Evolution of Unstable Quantum States and a Resolution of Zeno's Paradox, Phys. Rev. D16, 520 (1977).

[10] K. Savvidou, The Action Operator in Continuous Time Histories, J. Math. Phys. 40, 5657, 1999.

[11] N. Savvidou, General Relativity Histories Theory: Spacetime Diffeomorphisms and the Dirac Algebra of Constraints, Class. Quant. Grav. 18, 3611, 2001.

[12] C. Anastopoulos, Quantum Processes on Phase Space, Ann. Phys. 303, 275 (2003).

[13] C. Anastopoulos, Quantum vs Stochastic Processes and the Role of Quantum Numbers, to appear in Int. J. Theor. Phys. (2003), gr-qc/0208031.

[14] C. J. Isham, Canonical Quantum Gravity and the Problem of Time, GIFT Seminar 1992, 0157, gr-qc/9210011.

[15] C. Kuchar, Time and Inerpretations of Quantum Gravity, General Relativity and Relativistic Astrophysics, Winnipeg 91 Proceedings, 211 (1991).

[16] C. Anastopoulos and N. Savvidou, Quantum Mechanical Histories and the Berry Phase, Int. J. Theor. Phys. 41, 529 (2002).

[17] A. Kent. Consistent Sets Yield Contradictory Inferences in Quantum Theory. Phys. Rev. Lett. 78: 2874, 1997.

[18] R. Griffiths and J. B. Hartle. Comment on "Consistent Sets Yield Contrary Inferences in Quantum Theory". Phys. Rev. Lett. 81: 1981, 1998.

[19] C. J. Isham. Topos Theory and Consistent Histories: the Internal Logic of the Set of All Consistent Sets. Int. J. Theor. Phys. 36: 785, 1997.

[20] K. Savvidou, Continuous Time in Consistent Histories, gr-qc/9912076.

[21] M. V. Berry, Quantal Phase Factors Accompanying Adiabatic Changes, Proc. Roy. Soc. Lond. A392,45 (1984).

[22] A. Shapere and F. Wilczek (editors), Geometric Phases in Physics, (World Scientific, Singapore, 1989).

[23] B. Simon. Holonomy, the Quantum Adiabatic Theorem and Berry's Phase. Phys. Rev. Lett. 51, 2167 (1983).

[24] S. Pancharatnam, , Proc. Ind. Acad. Sci. A 44, 246 (1956) . 
[25] Y. Aharonov and J. Anandan. Phase Change During a Cyclic Quantum Evolution, Phys. Rev. Lett. 58, 1593 (1987).

[26] C. Anastopoulos, Continuous-time Histories: Observables, Probabilities, Phase Space Structure and the Classical Limit, J.Math.Phys. 42, 3225 (2001).

[27] C. Anastopoulos, Quantum Theory without Hilbert Spaces, Found. Phys. 31,1545 (2001).

[28] J. S. Schwinger,Brownian Motion of a Quantum Oscillator, J. Math. Phys. 2, 407 (1961).

[29] D. N. Page, Geometrical Description of Berry's Phase, Phys. Rev. A36, 3479 (1987).

[30] J. R. Klauder and B. Skagerstam (editors), Coherent States: Applications in Physics and Mathematical Physics, (World Scientific, Singapore, 1985).

[31] R. Omnés, Logical Reformulation of Quantum Mechanics: 4. Projectors in Semiclassical Physics, J. Stat. Phys. 57, 357, 1989.

[32] A. P. Balachandran and A. M. Roy, A Quantum Anti-Zeno Paradox, Phys. Rev. Lett. 84, 4019 (2000).

[33] V.V. Dodonov. E. V. Kurmyshev, V. I. Manko, Generalized Uncertainty Relation and Correlated Coherent States, Phys. Lett. A79, 150 (1980).

[34] G. C. Ghirardi, C. Omero, T. Weber and A. Rimini, Small Time Behaviour of Quantum Non-Decay Probability and Zeno's Paradox in Quantum Mechanics, Nuovo Cim. 52A, 421 (1979).

[35] S. Kochen and R. P. Specker, The Problem of Hidden Variables in Quantum Mechanics, J. Math. Mech. 17, 59 (1967).

[36] J. R. Klauder, Quantization is Geometry, After All, Ann. Phys. 188, 120, 1988; Geometric Quantization from a Coherent State Viewpoint, quant$\mathrm{ph} / 9510008$.

[37] J. R. Klauder and P. Maraner, Dynamics as Shadow of Phase Space Geometry, Ann. Phys. 253, 356 (1997). 8

[38] C.J.Isham and N. Linden, Continuous Histories and the History Group in Generalised Quantum Theory, J. Math. Phys. 36, 5392 (1995).

[39] C. Isham, N. Linden, K. Savvidou and S. Schreckenberg, Continuous Time and Consistent Histories, J. Math. Phys. 37, 2261 (1998).

[40] N. Savvidou, Poincaré Invariance for Continuous-Time Histories, J. Math. Phys. 43, 3053 (2002). 
[41] J. B. Hartle, Time and Time-Functions in Parametrized Non-relativistic Quantum Mechanics, Class. Quant. Grav. 13, 361 (1996).

[42] K. Savvidou and C. Anastopoulos, Histories Quantization of Parameterized Systems: Development of a General Algorithm, Class. Quant. Grav. 17, 2463 (2000).

[43] T. D. Newton and E. P. Wigner, Localised States for Elementary Systems, Rev. Mod. Phys. 21, 400 (1949).

[44] N. Savvidou, General Relativity Histories: Symmetries, to appear. 